\section{Bioseguridad en la atención odontológica a partir de la pandemia COVID-19: un análisis global de las nuevas medidas}

\author{
Biosecurity in dental care for the \\ COVID-19 pandemic: a global analysis of \\ the new measures
}

\section{Resumen}

Luego del brote de COVID-19 en el mundo, en medio de una crisis sanitaria, la odontología como profesión de alto nivel de riesgo de contaminación se plantea un reto, para la restructuración de sus procedimientos. $\mathrm{Al}$ respecto, se han revisado diferentes enfoques de las guías internacionales con diferentes realidades y experiencias en el campo, llegando a recomendaciones para el diseńo y uso del óptimo equipo de protección personal (EPP) en tiempos de escasez, minimizando los riesgos de contaminación. Sin embargo, la mayor parte de estas no están apoyadas con evidencia científica odontológica, mientras que se van desarrollando nuevos dispositivos, minimizando la exposición a procedimientos generadores de aerosoles (PGA) como barreras físicas.

Palabras clave: SARS-CoV-2; Equipo de protección personal; Coronavirus; COVID-19; Procedimientos generadores de aerosoles (fuente: DeCS BIREME).

\begin{abstract}
After the outbreak of COVID-19 in the world, during a health crisis, dentistry as a profession with a high level of risk of contamination poses a challenge, for the restructuring of its procedures. In this regard, different approaches to international guidelines have been reviewed with diverse realities and experiences in the field, reaching recommendations for the design and use of optimal personal protective equipment (PPE) in times of scarcity, minimizing the risks of contamination. However, most of these are not supported by dental scientific evidence, while new devices are being developed, minimizing exposure to aerosol-generating procedures (PGA) as physical barriers.
\end{abstract}

Keywords: SARS-CoV-2; Personal protective equipment; Coronavirus; COVID-19; Aerosol generating procedures (source: MeSH NLM).

\section{Artículo de Revisión}

Percy Romero Tapia 1,2,a , Jorge Eduardo Marin Aliaga ${ }^{1,2, b}$, Gabriela Sedano Balbín 2,b

${ }^{1}$ Hospital Nacional Guillermo Almenara Irigoyen, EsSalud Lima, Perú.

2 Universidad Nacional Mayor de San Marcos, Facultad de Odontología, Lima, Perú.

a Especialista en Cirugía Buco Maxilofacial.

${ }^{\mathrm{b}}$ Cirujano Dentista.

\section{Correspondencia:}

Percy Romero Tapia: drpercyromero@gmail.com Av. José Pardo 182- Dpto. 302. Miraflores, Lima, Perú. ORCID: 0000-0003-1383-5800

\section{Coautores:}

José Eduardo Marín Aliaga: drjorgeduardomarin@gmail. com

ORCID ID: 0000-0002-0061-7209

Gabriela Sedano Balbín: gsedanob@unmsm.edu.pe ORCID ID: 0000-0003-3811-8437.

\section{Editor:}

Juan Carlos Cuevas-González

Universidad Autónoma de Ciudad Juárez, México.

Conflicto de intereses: los autores declaran no tener conflictos de interés.

\section{Fuente de financiamiento: sin financiamiento.}

Recibido: $21 / 11 / 20$

Aceptado: $15 / 12 / 20$

Publicado: $15 / 02 / 21$ 


\section{Introducción}

Luego de la experiencia de dos epidemias de Beta coronavirus, el síndrome respiratorio agudo severo (SARS$\mathrm{CoV})^{1}$ y el síndrome respiratorio del medio oriente (MERS-CoV) ${ }^{2}$, se identificó el potencial infeccioso por la alta mortalidad e ingresos a Unidad de Cuidados Intensivos (UCI) de pacientes con diagnóstico de SARS-CoV2 ${ }^{3}$, siendo en la actualidad una pandemia que está en proceso de estudio y de la cual aprendemos cada día más.

La Organización Mundial de la Salud (OMS) estableció que las políticas de salud pública y privadas en relación a la atención odontológica, deberían de reestructurarse tomando nuevas medidas, tales como: un estricto triaje, el seguimiento de los casos confirmados del personal de atención y de los pacientes, la implementación de nueva infraestructura, el respeto al distanciamiento social, la descontinuación de la atención rutinaria ${ }^{4}$, la limitación a atenciones de emergencias y urgencias y la minimización de los procedimientos generadores de aerosoles (PGA) como el uso de la turbina ${ }^{5}$. Paralelamente las guías americanas y europeas de los Centros de Control y Prevención de Enfermedades (CDC), sugirieron que la atención odontológica de emergencias y urgencias, no debería descontinuarse y debería realizarse de manera inmediata durante la pandemia. para evitar daños colaterales a los pacientes sintomáticos ${ }^{6}$.

La Agencia Americana de la Administración de Seguridad y Salud Ocupacional (OSHA) incluyó a los odontólogos entre los profesionales con muy alto riesgo de exposición al SARS-CoV-2, sugiriendo controles de triaje al personal y a los pacientes, además del uso adecuado de EPP, debido al alto riesgo de contagio durante los
$\mathrm{PGA}^{7}$. Por su parte el Ministerio de Salud (MINSA) de Perú, recomendó en todos los niveles de atención y en las Unidades Prestadoras de Servicios de Salud, el uso de EPP completo con respiradores y protectores faciales con pantalla fija ${ }^{8}$; sin embargo, está recomendación de primera intención no incluía el uso de EPP en la toma de radiografías panorámicas ${ }^{9}$.

\section{Modo de transmisión viral: tamaño de las partículas}

Las principales formas de propagación de SARS-CoV-2 ocurren a través del contacto por gotitas transmitidas en el aire, por fómites contaminados y por PGA en pacientes prodrómicos y asintomaticos ${ }^{10}$. Estas gotitas respiratorias (de 5 a $10 \mu \mathrm{m}$ de diámetro) y los aerosoles (menores de $5 \mu \mathrm{m}$ ), quedan suspendidos en el aire con presencia de ARN de SARS-CoV-2 con capacidad de replicación de entre 3 a 16 horas ${ }^{11-13}$ (Figura 1).

El potencial infeccioso del SARS-CoV-2 está en el tamaño de las partículas transportadas por las personas infectadas, de una masa media de $2 \mu \mathrm{m}$, las cuales son respirables a distancias cortas en forma de bioaerosoles ${ }^{13}$. Estudios previos realizados en macacos Rhesus, sostienen que las células epiteliales de la enzima convertidora de angiotensina (ECA2), que recubren los conductos de las glándulas salivales de la laringofaringe, eran el objetivo primario para el SARS-CoV-1 y de allí su potencial de dispersión a través de la saliva ${ }^{14}$. Por tales motivos, la OMS recomendó el uso de enjuagues bucales con peróxido de hidrogeno $1 \%$ y/o povidona yodada $0,2 \%$ para disminuir el potencial infeccioso del SARS-CoV-2; sin embargo, en la actualidad no existe suficiente evidencia que apoye tal recomendación ${ }^{4}$.

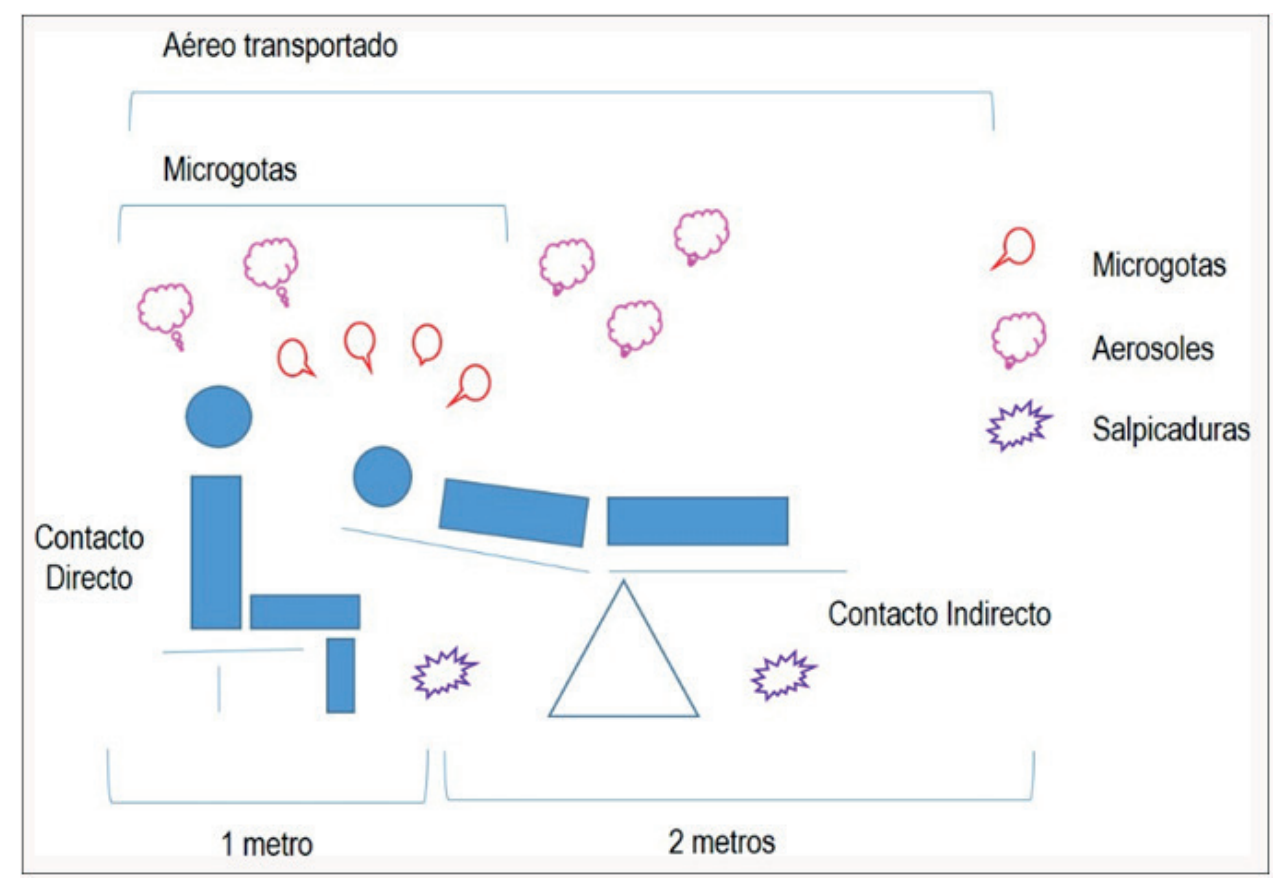

Figura 1. Ilustración de rangos de tamaño de partículas y rutas de infección cruzada en la atención odontológica durante los PGA ${ }^{15}$ 


\section{Medidas implementadas en la atención odontológica a partir de la pandemia COVID-19}

Los hospitales en el epicentro de Wuhan continuaron con las atenciones odontológicas, priorizando emergencias y urgencias hasta febrero del presente año, implementando triaje con encuestas virtuales, realizando tratamientos quirúrgicos en consultorio mediante el uso del EPP y las barreras de bioseguridad ${ }^{15,16}$, teniendo en consideración la limitación de los procedimientos invasivos y la toma imagenológica extraoral ${ }^{15}$, el uso de enjuagues bucales previo a los PGA con succión de alto volumen y el uso de diques de goma ${ }^{16,17}$.

En el centro de la pandemia en un hospital de Wuhan se tuvo consideraciones de triaje diferenciado vía telefónica y física, incidiendo en preguntas sobre estado actual de salud e historial de viajes ${ }^{16}$, y defirieron los tratamientos hasta 1 mes después, imitando lo considerado en la epidemia del SARS-CoV ${ }^{18}$. Por otro lado, el CDC sugirió la interrupción del aislamiento para pacientes confirmados en entorno domiciliario y en asintomáticos con positividad del SARS-CoV-2 en RT- PCR, después de 10 días de haberse iniciado los síntomas, además de 24 horas de ausencia de fiebre sin necesidad de antipiréticos ${ }^{20}$. Pese a ello, no se pudo estimar la cantidad de brecha de espera a la atención después de la primera infección por el SARS-CoV-2.

A nivel mundial se han desarrollado varias guías de práctica clínica y de recomendaciones, acerca de la implementación de medidas en la atención odontológica en tiempos de pandemia. En el Perú se han emitido directivas a través del MINSA, indicando la necesidad del uso de EPP, lentes y/o protectores faciales, guantes, mandilón descartable ${ }^{20}$; recomendando el indispensable uso de respiradores N95, FFP2 (filtering face piece: respiradores o mascarillas filtrantes) o superiores en los PGA ${ }^{21,22}$, limitando la atención a emergencias y urgencias ${ }^{23}$, apoyándose en el enfoque de la ADA (Asociación Dental Americana) ${ }^{24}$, publicado en su boletín para pacientes meses después del inicio de la pandemia ${ }^{25}$.

De manera global la reapertura de los servicios odontológicos fue evaluada en 11 países hasta Setiembre del 2020, apoyándose en las guías de los CDC y la ADA, sugiriendo que los PGA, tales como: uso de piezas de mano de alta y baja velocidad, raspadores ultrasónicos, jeringas de agua/aire, toma de radiografías intraorales, constituyen un alto riesgo de infección. Al mismo tiempo implementando nuevas medidas de atención, como el triaje telefónico mediante encuestas, acondicionamiento del consultorio, protocolos de toma de temperatura al ingreso, el no uso de formatos impresos, adecuado uso de EPP, cambios de respiradores por paciente, sólo un par de guantes estériles y el uso de protectores faciales en casos confirmados ${ }^{26}$. Adicionalmente, el uso del EPP antes de ingresar al área de procedimientos, restringiendo el uso de la escupidera, uso de diques de goma, succión de alto nivel y el uso de enjuagues bucales ${ }^{26}$, con espacios de tiempo entre pacientes de 2 a 180 minutos, y en casos confirmados de hasta 3 horas ${ }^{27}$, además de ambientes acondicionados previamente con HEPA en un cuarto de aislamiento para casos confirmados ${ }^{26}$.

\section{Nivel apropiado de equipo de protección personal (EPP)}

La OMS sugiere que el personal de salud y el personal de limpieza expuestos a los PGA en la atención de pacientes COVID-19, debería realizarla con respiradores N95 o FFP2, estándar o el equivalente ${ }^{28}$. Sin embargo, debido a la escasez mundial, recomendaron la implementación de espacios laborales con barreras físicas, solo para casos de emergencias y urgencias, justificando el uso de doble guantes para los casos quirúrgicos, desaconsejando la reutilización de respiradores o cualquier parte del EPP sin previa descontaminación y esterilización ${ }^{29}$, limitando el uso de respiradores a PGA, tales como: las traqueotomías e intubaciones endotraqueales ${ }^{30}$, pero no al uso de piezas de mano en la atención odontológica que son una fuente conocida de generación de aerosoles.

Existe un mayor riesgo de filtración y contaminación durante la exposición de los PGA, como en las zonas del cuello, antebrazos, muñecas y manos ${ }^{31}$, por lo que cubrir más partes del cuerpo, obtiene mayor protección, ante salpicaduras y microgotas; sin embargo, también existe el riesgo de auto contaminación al retiro de los EPP, por lo que el uso de mangas selladas con guantes, podría ser una ayuda ante la dificultad de la colocación y el retiro del EPP. Por otro lado, el uso de dobles guantes y su retiro en un solo paso conjuntamente con la bata, a la evaluación de marcadores fluorescentes, han demostrado un menor riesgo de auto contaminación, según las recomendaciones de los $\mathrm{CDC}^{32}$.

Para la atención rutinaria de los pacientes sospechosos o confirmados, los CDC sugieren el uso adecuado de EPP ${ }^{35}$, además de la implementación de salas de aislamiento con filtros de aire de partículas de alta eficiencia (HEPA), junto al uso de respiradores N95, o superiores, aprobados por el Instituto Nacional para la Seguridad y Salud Ocupacional (NIOSH) en los Estados Unidos. Con énfasis en el uso de estos dispositivos por un tiempo no mayor de 8 a 12 horas ${ }^{34}$, y en tiempos de escasez recomiendan el uso de batas de tela reutilizables u overoles con cobertura a los $360^{\circ}{ }^{33}$, junto a respiradores purificadores de aire elastoméricos 34 . Éstos últimos disponen de filtros con niveles desde el 95 al 100\%, logrando un sellado adecuado, igual al de los N95, efectivizando el filtro de aire, aunque contraindicados en áreas quirúrgicas por no filtrar el aire exhalado y el potencial riesgo de contaminación cruzada $^{35}$ (Figura 2).

\section{Estrategias diseñadas para reducir el nivel de contaminación durante los PGA}

Las guías clínicas para los PGA desarrolladas en 62 países, además de la Directiva Sanitaria $\mathrm{N}^{\circ} 100 / \mathrm{MIN}$ SA/2020/DGIESP acerca del manejo de la atención estomatológica en Perú, sugieren realizar los PGA en 


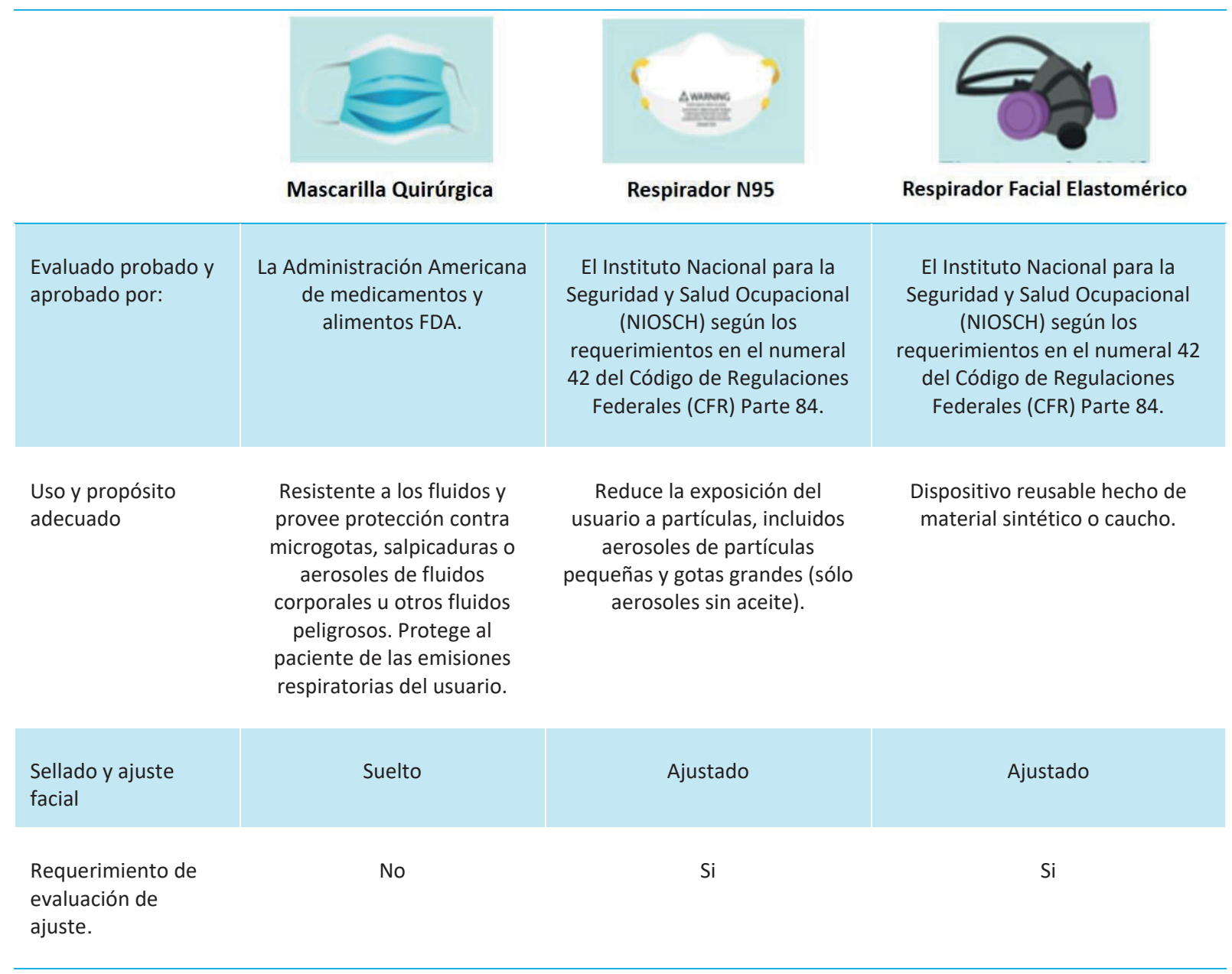

Figura 2. Diferencia entre los tipos de mascarillas según los CDCs ${ }^{36}$

áreas hospitalarias o especializadas, con un nivel superior de EPP en pacientes confirmados, el uso de enjuagues preoperatorios con $\mathrm{H} 2 \mathrm{O} 2$, povidona yodada, cloruro de cetilperidinio, para minimizar los aerosoles biológicos ${ }^{27}$. De manera opcional, el uso de la clorhexidina como enjuague oral, debido a que reduce hasta un $64,8 \%$ las unidades formadoras de colonias (UFC) en los PGA ${ }^{37}$.

Con el objetivo de optimizar las barreras de bioseguridad, se han desarrollado dispositivos en el área de atención hospitalaria para protegerse de la dispersión de los PGA, denominándolas "Caja de Aerosol", diseñadas en un primer momento para su empleo en anestesiología durante la intubación ${ }^{38,39}$, teniendo modificaciones como cámaras de aislamiento de plástico transparentes con tubos de polivinilo en su estructura soporte ${ }^{40}$, mientras que otros diseños optaron por estructuras de policarbonato transparente aplicadas en cardiocirugía y también anestesiología ${ }^{41,42}$ (Figura 3).

Finalmente se han reportado dispositivos adaptados a la odontología fabricados a base de acrílicos translucidos adheridos a la unidad odontológica con conexiones para aspiración y filtro de aire a presión negativa que se evaluaron junto a el uso de marcadores fluorescentes, simulando procedimientos odontológicos a través del uso de un dispositivo efectivo que serviría como barrera aunque aún sin validar su efectividad para evitar el contagio ${ }^{43}$ (Figura 4).

\section{Conclusiones}

La atención odontológica tiene un alto riesgo de infección cruzada entre el personal de atención y el paciente en la atención de pacientes confirmados o sospechosos con COVID-19, por la proximidad de estos y los PGA, provenientes de los dispositivos odontológicos, como la pieza de mano de alta y baja velocidad y el uso de ultrasonido; por tal motivo el uso del EPP es una forma de minimizar la exposición a los fluidos corporales contaminados, evitando que salpicaduras, micro gotas, o aerosoles, puedan llegar a superficies corporales o ingresar a través de mucosas orales, nasales u oculares.

Se han brindado múltiples sugerencias y recomendaciones en las guías internacionales y nacionales, sin embargo, muchas de éstas no están apoyadas en evidencia científica, por lo que se alienta a desarrollar más estudios dirigidos a la atención odontológica, tanto en consulta externa como en sala de operaciones. 


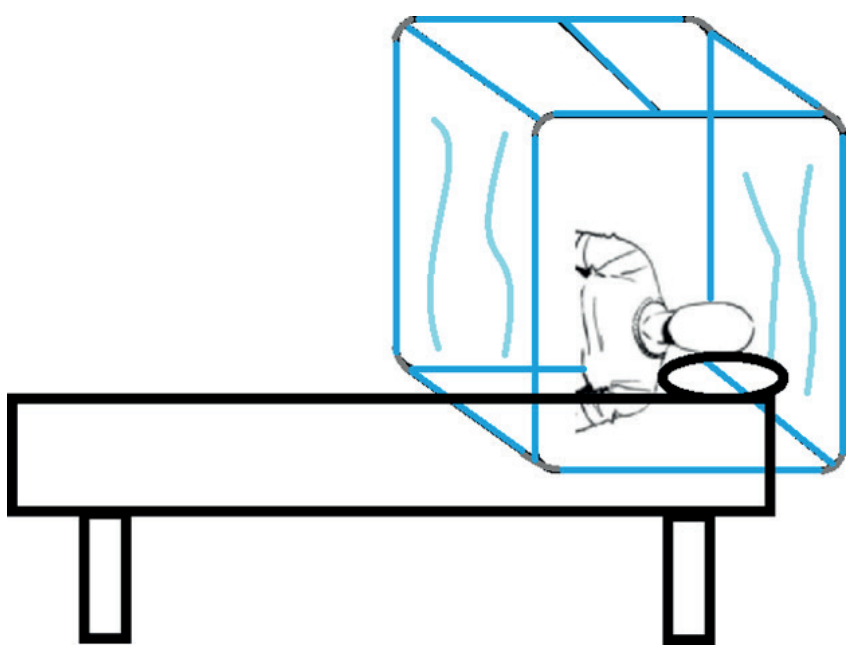

Figura 3. Representación de dispositivo adaptado a sala de operaciones para la intubación ${ }^{40}$

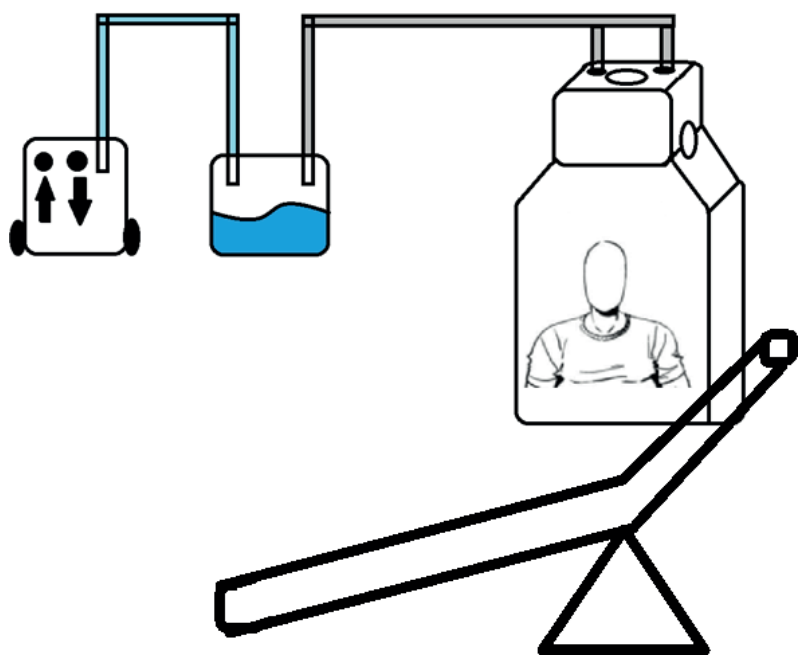

Figura 4. Representación esquemática de dispositivo barrera para PGA adaptado a la atención odontológica ${ }^{43}$

\section{Referencias bibliográficas.}

1. Ksiazek TG, Erdman D, Goldsmith CS, et al. A novel coronavirus associated with severe acute respiratory syndrome. N Engl J Med. 2003;348:1953-66.

2. de Groot RJ, Baker SC, Baric RS, et al. Middle East Respiratory Syndrome Coronavirus (MERS-CoV): Announcement of the Coronavirus Study Group. J Virol. 2013;87(14):7790-7792.

3. Huang C, Wang Y, Li X, et al. Clinical features of patients infected with 2019 novel coronavirus in Wuhan, China. Lancet. 2020;395(10223):497-506.

4. World Health Organization (WHO). Considerations for the Provision of Essential Oral Health Services in the Context of COVID-19: interim guidance. WHO. 2020, No. WHO/2019-nCoV/Oral_health/2020.1:1-5.

5. World Health Organization (WHO). Maintaining essential health services: operational guidance for the COVID-19 context: interim guidance. WHO. 2020, No. WHO/2019-nCoV/essential_health_services/2020.2:1-61.
6. Centers for Disease Control And Prevention [sede Web]. USA: CDC. 2020-[actualizada el 30 de Junio del 2020; acceso 12 de setiembre de 2020] CDC; Framework for Healthcare Systems Providing Non- COVID-19 Clinical Care During the COVID-19 Pandemic [aproximadamente 3 páginas] Disponible en: https://www.cdc. gov/coronavirus/2019-ncov/hcp/framework-non-COVID-care.html.

7. Occupational Safety and Health Administration. US Department of Labor. Guidance on Preparing Workplaces for COVID-19. OSHA 3990-03 2020. Disponible en: https://www.osha.gov/Publications/OSHA3990.pdf.

8. Documento Técnico: Recomendaciones Sobre El Uso de Escudos Faciales (Caretas) En Los Establecimientos de Salud y En La Comunidad En El Contexto de La Pandemia de COVID-19. Resolución Ministerial N ${ }^{\circ} 447$ 2020/MINSA, 30 de Junio del 2020.

9. Norma Técnica de salud para el uso de Los equipos de protección personal por los trabajadores de las instituciones prestadores de servicio de salud. Resolución Ministerial N 456-2020/MINSA, 02 de Julio del 2020. 
10. World Health Organization (WHO). Transmission of SARS-CoV-2: implications for infection prevention precautions. WHO.2020, No. WHO/2019-nCoV/Sci_ Brief/Transmission_modes/2020.3:1-10.

11. World Health Organization (WHO). Infection prevention and control of epidemic- and pandemic-prone acute respiratory infections in healthcare. WHO. 2014:1-156.

12. van Doremalen N, Bushmaker T, Morris DH, et al. Aerosol and surface stability of SARS-CoV-2 as compared with SARS-CoV-1. N Eng J Med. 2020;382(16):156467.

13. Fears AC, Klimstra WB, Duprex P, et al. Persistence of Severe Acute Respiratory Syndrome Coronavirus 2 in Aerosol Suspensions. Emerg Infect Dis. 2020;26(9). 2168-71

14. Liu L, Wei Q, Alvarez X, et al. Epithelial cells lining salivary gland ducts are early target cells of severe acute respiratory syndrome coronavirus infection in the upper respiratory tracts of rhesus macaques. J Virol. 2011;85:4025-30.

15. Short Life Working Group (SLWG). Ventilation, Water and Environmental Cleaning in Dental Surgeries Relating to COVID-19. Scotland National Services. 2020:182

16. Meng L, Hua F, Bian Z. Coronavirus Disease 2019 (COVID-19): Emerging and Future Challenges for Dental and Oral Medicine. J Dent Res. 2020;99(5):481-87.

17. Li ZY, Meng LY. [The prevention and control of new coronavirus infection in department of stomatology]. Zhonghua Kou Qiang Yi Xue Za Zhi. 2020;55(0):E001. Chinese.

18. Harrel SK, Molinari J. Aerosols and splatter in dentistry: A brief review of the literature and infection control implications. J Am Dent Assoc. 2004;135(4):429-37.

19. Samaranayake LP, Peiris M. Severe acute respiratory syndrome and dentistry A retrospective view. J Am Dent Assoc. 2004;135:1292-302.

20. Centers for Disease Control And Prevention [sede Web]. USA: CDC. 2020- [actualizada el 20 de Julio del 2020; acceso 12 de setiembre de 2020] CDC; Discontinuation of Isolation for Persons with COVID-19 Not in Healthcare Settings; [aproximadamente 5 páginas]. Disponible en: https://www.cdc.gov/coronavirus/2019-ncov/hcp/ disposition-in-home-patients.html\#: : :text=Persons $\% 20$ with\%20COVID\%2D19\%20who ,of\%20fever\%2Dreducing\%20medications\%20and.

21. Documento Técnico: Prevención, Diagnóstico y Tratamiento de Personas Afectadas Por COVID-19 En El Perú. Resolución Ministerial N 193-2020/MINSA, 13 de Abril del 2020

22. Documento Técnico: Recomendaciones para el uso apropiado de mascarillas y respiradores por el personal de salud en el contexto del COVID-19. Resolución Ministerial N² 248-2020/MINSA, 03 de Mayo del 2020.

23. Modificatoria del Documento Técnico: Recomendaciones para el Uso Apropiado de Mascarillas y Respiradores por el Personal de Salud en el Contexto del COVID-19. Resolución Ministerial No 268-2020/MINSA, 07 de Mayo del 2020.
24. Directiva Sanitaria Nº100/Minsa/2020/DGIESP-Manejo de la Atención Estomatológica en el contexto de la Pandemia por COVID-19. Resolución Ministerial $\mathrm{N}^{\circ}$ 288-2020/MINSA,16 de Mayo del 2020.

25. American Dental Association [sede Web]. USA: ADA. 2020 [actualizada el 31 de Marzo del 2020; acceso 12 de setiembre de 2020] What Constitutes a Dental Emergency?; [1 página]. Disponible en: https://success.ada.org/ / media/CPS/Files/Open $\% 20$ Files/ADA_COVID19_Dental_Emergency_DDS. pdf?_ga $=2.253879752 .110187285 .1584496315$ 1622146531.1565271894 .

26. American Dental Association [sede Web]. USA: ADA. 2020 [actualizada el 19 de Marzo del 2020; acceso 12 de setiembre de 2020] What Is a Dental Emergency?; [1 página]. Disponible en: https://www.ada.org/ //media/ CPS/Files/COVID/ADA_DentalEmerg_Patient_Flyer. pdf?utm_source=adaorg\&utm_medium $=$ covid-resources-lp\&utm_content=cv-pm-emerg-def-patients\&utm_ campaign $=$ covid -19 .

27. Clarkson J, Ramsay C, Aceves M. Recommendations for the re-opening of dental services: a rapid review of international sources. Cochrane Oral Health. 2020:1-36.

28. Clarkson J, Ramsay C, Richards D, et al. Aerosol Generating Procedures and their Mitigation in International Dental Guidance Documents - A Rapid Review. Cochrane Oral Health. 2020:1-69.

29. World Health Organization (WHO). Rational use of personal protective equipment for coronavirus disease (COVID-19) and considerations during severe shortages Interim guidance. WHO, 2020, No. WHO/2019nCov/IPC_PPE_use/2020.3:1-28.

30. World Health Organization (WHO). Advice on the Use of Masks in the Context of COVID-19: Interim Guidance-2. WHO, 2020. No. WHO/2019-nCov/IPC_ Masks/2020.4:1-16.

31. Zamora JE, Murdoch J, Simchison B, Day AG. Contamination: A comparison of 2 personal protective systems. Can Med Assoc. 2006;175(3):249- 54.

32. Verbeek JH, Rajamaki B, Ijaz S, et al. Personal protective equipment for preventing highly infectious diseases due to exposure to contaminated body fluids in healthcare staff. Cochrane Database Syst Rev. 2020;5:1-147.

33. Centers for Disease Control And Prevention [sede Web]. USA: CDC. 2020- [actualizada el 20 de Julio del 2020; acceso 12 de setiembre de 2020] CDC; Strategies for Optimizing the Supply of Isolation Gowns; [aproximadamente 5 páginas]. Disponible en: https://www. cdc.gov/coronavirus/2019-ncov/hcp/ppe-strategy/isolation-gowns.html.

34. Centers for Disease Control And Prevention [sede Web]. USA: CDC. 2020-[actualizada el 28 de Junio del 2020; acceso 12 de setiembre de 2020] CDC; Strategies for Optimizing the Supply of N95 Respirators Personal Protective Equipment Respiratory Protection [aproximadamente 5 páginas] Disponible en: https://www.cdc.gov/ coronavirus/2019-ncov/hcp/respirators-strategy/index. html.

35. Centers for Disease Control And Prevention [sede Web]. USA: CDC. 2020- [actualizada el 13 de Octubre del 
2020; acceso 12 de setiembre de 2020] CDC; Elastomeric Respirators: Strategies During Conventional and Surge Demand Situations Conventional,Contingency, and Crisis Strategies; [aproximadamente 4 páginas] Disponible en: https://www.cdc.gov/coronavirus/2019-ncov/hcp/ elastomeric-respirators- strategy/index.html.

36. Centers for Disease Control and Prevention [sede Web]. USA: CDC. 2020- [actualizada el 02 de Julio del 2018; acceso 12 de setiembre de 2020] Understanding the Difference; [1 página]. Disponible en: https://www.cdc.gov/ niosh/npptl/pdfs/UnderstandingDifference3-508.pdf.

37. Marui VC, Souto MLS, Rovai ES, et al. Efficacy of preprocedural mouthrinses in the reduction of microorganisms in aerosol: A systematic review. J Am Dent Assoc. 2019;150(12):1015-26.

38. Canelli Robert Connor CW, Gonzalez M, et al. Barrier enclosure during endotracheal intubation. $\mathrm{N}$ Engl J Med. 2020;382(20):1-2.

39. https://www.taiwannews.com.tw/Keoni E. Taiwanese doctor invents device to protect US doctors against coronaviruse. EEUU; 2020- [actualizada el 23 de Marzo del 2020; acceso 14 de setiembre de 2020] Disponible en: https:/www.taiwannews.com.tw/en/news/3902435.
40. Cubillos J, Querney J, Rankin A, et al. A multipurpose portable negative air flow isolation chamber for aerosol-generating procedures during the COVID-19 pandemic. Br J Anaesth. 2020;125(1):179-81.

41. Leyva Moraga FA, Leyva Moraga E, Leyva Moraga F, et al. Aerosol box, An Operating Room Security Measure in COVID-19 Pandemic. World J Surg. 2020;44(7):2049-50.

42. Girgis AM, Merna NA, Tilvawala CG, et al. Novel Coronavirus Disease 2019 (COVID-19) Aerosolization Box: Design Modifications for Patient Safety. J Cardiothorac Vasc Anesth. 2020;34:2274-86.

43. Teichert-Filho R, Baldasso CN, Campos MM, et al. Protective device to reduce aerosol dispersion in dental care in times of COVID-19 pandemic. Int Endod J. 2020;11:1588-97. 
